\title{
REPRESENTATION OF MODULAR LATTICES AND OF RELATION ALGEBRAS
}

\author{
BY \\ BJARNI JÓNSSON
}

Introduction. This paper gives an axiomatic characterization of the class of all those (modular) lattices which are isomorphic to lattices of commuting equivalence relations. As night be expected, this problem turns out to be closely related to the representation problem for relation algebras, and we are able to borrow some basic ideas from the work of R. Lyndon $[7 ; 8]$. It turns out to be convenient to consider first the class of all those algebras $\mathfrak{A}$

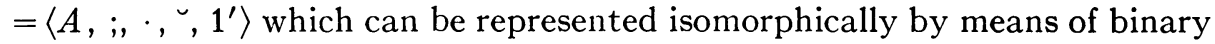
relations over some set $U$ in such a way that the operations ;, ., “ correspond to relation-theoretic multiplication, set-theoretic multiplication and relationtheoretic conversion, respectively, and $1^{\prime}$ corresponds to the identity relation over $U$. This class of algebras is characterized in Theorem 1. In Theorem 2 this result is applied to lattices. The key observation here is that a lattice $\mathfrak{N}=\langle A,+, \cdot\rangle$ with a zero element 0 is isomorphic to a lattice of commuting

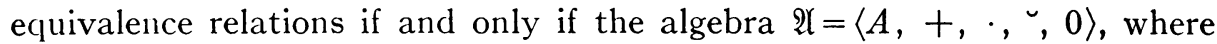

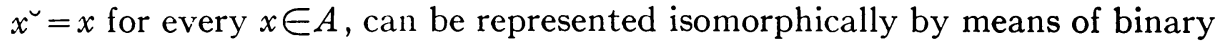
relations in the manner discussed above with + and 0 taking the place of ; and $1^{\prime}$.

We can give a more direct, although more involved, proof of Theorem 2 . The added complications are due mostly to the fact that we are then unable to take advantage of the metamathematical results of Henkin [2] and Tarski [9]. The trouble is that we see no direct way of proving that the class of lattices under consideration coincides with the class of all subalgebras of algebras from an arithmetic class in the wider sense. The method employed here also has the advantage that it brings out the close connection with the representation problem for relation algebras, and in this context Theorem 1 is of independent interest. Theorems 3 and 4 concern the existence of what we call weak representations for relation algebras, and the paper concludes with a brief discussion of some open questions related to our work.

1. Preliminaries. We adopt the notation and terminology of Tarski [9], in particular, we refer the reader to that paper for the definitions of an arithmetic class and an arithmetic class in the wider sense. The relationtheoretic product of two binary relations $R$ and $S$ will be denoted by $R \mid S$, their set-theoretic sum and product by $R \cup S$ and $R \cap S$, and the relationtheoretic converse of $R$ by $R^{-1}$. By an algebra of relations we mean a system $\langle Q, \mid, \cap,-1, I\rangle$ where $Q$ is a set of binary relations which is closed under the

Received by the editors, March 5, 1958. 
operations $\mid, \cap$ and $^{-1}, I \in Q$, and $R \mid I=R$ for every $R \in Q$. (Observe that this is a more general notion than the concept of a proper relation algebra.) We could actually, without any essential loss of generality, restrict ourselves to the case in which $I$ is the identity relation over some set $U$ and all the members of $Q$ are relations over $U$. In fact, if $\langle a, \mid, \cap,-1, I\rangle$ is an algebra of relations, then $I^{-1} \mid I=I$, whence it follows that $I$ is an equivalence relation over some set $V$. Since, for every $R \in Q, R \mid I=R$ and $R^{-1} \mid I=R^{-1}$, all the members of $Q$ are relations over $V$. Choose a function $f$ which maps $V$ onto some set $U$ in such a way that, for every $x, y \in V, f(x)=f(y)$ if and only if $\langle x, y\rangle \in I$, and for each $R \in Q$ let $F(R)$ be the relation consisting of all ordered pairs which are of the form $\langle f(x), f(y)\rangle$ for some $\langle x, y\rangle \in R$. It is easy to check that this function $F$ maps the given algebra of relations isomorphically onto an algebra of relations $\langle\otimes, \mid, \cap,-1, J\rangle$ where $J=F(I)$ is the identity relation over $U$. (An analogous result for proper relation algebras is found in JónssonTarski [6, Theorem 4.27].)

We shall be concerned with algebras consisting of a set $A$ and certain operations one of which will be denoted by the symbol $\cdot$. Since we will be primarily concerned with algebras which have, among other things, the property that $A$ is a semi-lattice under the operation $\cdot$, i.e. that

$$
a \cdot(b \cdot c)=(a \cdot b) \cdot c, \quad a \cdot b=b \cdot a \quad \text { and } a \cdot a=a
$$

for every $a, b, c \in A$, we introduce certain notations and terms suggested by this special case. The operation $\Pi$, acting on finite sequences of elements of $A$ is defined by the familiar formulas

$$
\prod_{k \leq 0} a_{k}=a_{0} \text { and } \prod_{k \leqq n+1} a_{k}=\left(\prod_{k \leq n} a_{k}\right) \cdot a_{n+1},
$$

and the relation $\leqq$ is defined by the condition that

$$
a \leqq b \text { if and only if } a \cdot b=a .
$$

An element $z \in A$ is called a zero element (of the algebra) if $z \leqq x$ for every $x \in A$, and an element $u \in A$ is called an atom (of the algebra) if $u$ is not a zero element and if, for every $x \in A$, the condition $x \leqq u$ implies that either $x=u$ or else $x$ is a zero element.

2. The main theorem. We now state our central result.

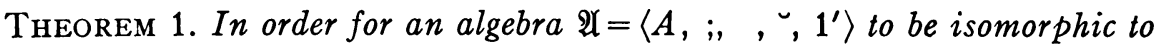
an algebra of relations it is necessary and sufficient that, for every $a, b, c \in A$,
(i) $a \cdot(b \cdot c)=(a \cdot b) \cdot c$,
(ii) $a \cdot b=b \cdot a$,
(iii) $a \cdot a=a$,
(iv) $a ;(b ; c)=(a ; b) ; c$,
(v) $a ; 1^{\prime}=a$,
(vi) $(a \cdot b) ; c \leqq b ; c$,
(vii) $a^{u}=a$,
(viii) $(a ; b)^{\llcorner}=b^{\llcorner} ; a^{\llcorner}$,
(ix) $(a \cdot b)^{\sim}=a^{\lrcorner} \cdot b^{\lrcorner}$,

and that the following condition be satisfied: 
(Г) Suppose $n$ is a positive integer, $a_{0}, a_{1}, \cdots, a_{2 n} \in A$ and, for each positive integer $k \leqq n, \phi(k)$ and $\psi(k)$ are natural numbers with $\phi(k) \leqq k$ and $\psi(k) \leqq k$. Let

$$
b_{1,0,1}=a_{0}, \quad b_{1,1,0}=a_{0}^{\check{0}}, \quad b_{1,0,0}=b_{1,1,1}=1^{\prime},
$$

and for $k=1,2, \cdots, n$ and $i, j=0,1, \cdots, k$ let

$\left(\Gamma_{2}\right) b_{k+1, i, j}=\prod_{p \leq k}\left(b_{k, i, p} ; b_{k, p j}\right)$,

( $\left.\Gamma_{3}\right) b_{k+1, i, k+1}=\left(b_{k+1, i, \phi(k)} ; a_{2 k-1}\right) \cdot\left(b_{k+1, i, \psi(k)} ; \widetilde{a_{2 k}}\right)$,

(Г⿻ $\left.\Gamma_{4}\right) b_{k+1, k+1, j}=\left(b_{k+1, j, k+1}\right)^{\swarrow}$,

( $\left.\Gamma_{5}\right) b_{k+1, k+1, k+1}=1^{\prime}$.

With these notations, if $b_{k, \phi(k), \psi(k)} \leqq a_{2 k-1} ; a_{2 k}$ for $k=1,2, \cdots, n$, then $a_{0}$ $\leqq b_{n+1,0,1}$.

The proof of Theorem 1 will be based on four lemmas.

Lemma A. The class of all algebras which are isomorphic to algebras of relations is an arithmetic class in the wider sense.

Proof. Let $K$ be the class of all algebras which are isomorphic to algebras of relations. According to Tarski [9, Theorem 1.13], it is sufficient to show that there exists an arithmetic class in the wider sense $L$ such that $K$ is the class of all sub-algebras of algebras belonging to $L$.

Consider an algebra $\mathfrak{A}=\left\langle A, ;, \cdot, \breve{1^{\prime}}\right\rangle$, and let $F$ be the function which associates with each $x \in A$ the relation consisting of all ordered pairs $\langle u, v\rangle$ such that $u$ and $v$ are atoms of $\mathfrak{Y}$ and $u \leqq x ; v$. We now define $L$ by the condition that $\mathfrak{A} \in L$ if and only if $F$ maps $\mathfrak{A}$ isomorphically onto an algebra of relations. Thus $\mathfrak{Q} \in L$ if and only if the following conditions are satisfied.

(1) $F$ is one-to-one.

(2) For every $x, y \in A, F(x ; y)=F(x) \mid F(y)$.

(3) For every $x, y \in A, F(x \cdot y)=F(x) \cap F(y)$.

(4) For every $x \in A, F\left(x^{4}\right)=F(x)^{-1}$.

(5) For every $x \in A, F(x) \mid F\left(1^{\prime}\right)=F(x)$.

It is easy to see that the conditions (1)-(5) are equivalent to the assertion that certain arithmetic formulae hold in $\mathfrak{A}$. In fact, (1) means that, for every $x, y \in A$,

$\left\{\begin{array}{l}\text { if the conditions } u \leqq x ; v \text { and } u \leqq y ; v \text { are equivalent for all atoms } \\ u, v \text { of } \mathfrak{A} \text {, then } x=y .\end{array}\right.$

Similarly, (2), (3), and (4) are equivalent to the assertion that, for all $x, y \in A$ and for all atoms $u, v$ of $\mathfrak{A}$,

(7) $u \leqq(x ; y) ; v$ if and only if $u \leqq x ; w$ and $w \leqq y ; v$ for some atom $w$ of $\mathfrak{A}$.

(8) $u \leqq(x \cdot y) ; v$ if and only if $u \leqq x ; v$ and $u \leqq y ; v$.

(9) $u \leqq x^{\sim} ; v$ if and only if $v \leqq x ; u$.

Finally, under the assumption that (1) and (2) hold, (5) is equivalent to the assertion that, for every $x \in A$, 
(10) $x ; 1^{\prime}=x$.

Thus the conditions (6)-(10) characterize $L$, and we conclude that $L$ is an arithmetic class.

Clearly every subalgebra of an algebra in $L$ belongs to $K$. In order to show that, conversely, every algebra in $K$ is a subalgebra of an algebra in $L$, we need only consider the case of an algebra of relations

$$
\mathfrak{A}=\left\langle\mathfrak{Q}, \mid, \cap,^{-1}, I\right\rangle .
$$

In view of a remark in $\S 1$, we may assume that $I$ is the identity relation over some set $U$, in which case every member of $Q$ is a relation over $U$. In this case $\mathfrak{A}$ is a subalgebra of the algebra

$$
\mathfrak{B}=\left\langle\mathbb{B}, \mid \cap,^{-1}, I\right\rangle
$$

where $B$ is the family consisting of all the binary relations over $U$.

$\mathfrak{B}$ has a zero element, namely the empty set $\varnothing$, and the atoms of $\mathfrak{B}$ are the relations in $\beta$ consisting of just one ordered pair. Suppose $\alpha, \beta \in U$ and let $P$ and $Q$ be the atoms of $\mathscr{B}$ whose sole members are $\langle\alpha, \beta\rangle$ and $\langle\beta, \beta\rangle$, respectively. For any $R \in B$ we then have $\langle\alpha, \beta\rangle \in R$ if and only if $P \subseteq R \mid Q$. Hence $\mathfrak{B}$ satisfies the condition (6). That (7) holds in $\mathscr{B}$ follows from the fact that every member of $B$ is a set-sum of atoms and that the operation $\mid$ is infinitely distributive with respect to set-addition. It is easy to check that

$$
(R \cap S) \mid Q=(R \mid Q) \cap(S \mid Q)
$$

whenever $R, S \in B$ and $Q$ is an atom of $\mathfrak{B}$, and this clearly implies that (8) holds. The four conditions

$$
P \subseteq R^{-1}\left|Q, \quad\left(R^{-1} \mid Q\right) \cap P \neq \varnothing, \quad(R \mid P) \cap Q \neq \varnothing, \quad Q \subseteq R\right| P
$$

are equivalent whenever $R \in \Theta$ and $P$ and $Q$ are atoms of $B$. Hence (9) holds. Since $\mathfrak{B}$ obviously satisfies (10), we conclude that $\mathfrak{B} \in L$, and the proof is complete.

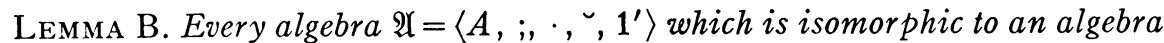
of relations satisfies the conditions (i)-(ix) and $(\Gamma)$.

Proof. That $\mathfrak{A}$ satisfies (i)-(ix) follows trivially from well-known identities in the calculus of binary relations. Assume now that the hypothesis of $(\Gamma)$ is satisfied. Let $F$ be a function which maps $\mathfrak{A}$ isomorphically onto an algebra of relations

$$
\langle Q, \mid, \cap,-1, I\rangle,
$$

and consider an ordered pair $\langle\alpha, \beta\rangle \in F\left(a_{0}\right)$. Letting $\gamma_{0}=\alpha$ and $\gamma_{1}=\beta$ we shall show that there exist elements $\gamma_{2}, \gamma_{3}, \cdots, \gamma_{n+1}$ such that if $1 \leqq k \leqq n+1$, then

$$
\left\langle\gamma_{i}, \gamma_{j}\right\rangle \in F\left(b_{k, i, j}\right) \quad \text { for } i, j=0,1, \cdots, k .
$$

By $\left(\Gamma_{1}\right),(1)$ holds for $k=1$. Assume that $1 \leqq q \leqq n$ and that $\gamma_{2}, \gamma_{3}, \cdots, \gamma_{q}$ 
have been chosen in such a way that (1) holds for $k=1,2, \cdots, q$. Applying (1) with $k=q$, we infer from $\left(\Gamma_{2}\right)$ that

$$
\left\langle\gamma_{i}, \gamma_{j}\right\rangle \in F\left(b_{q+1, i, j}\right) \quad \text { for } i, j=0,1, \cdots, q .
$$

Since, by our hypothesis,

$$
b_{q, \phi(q), \psi(q)} \leqq a_{2 q-1} ; a_{2 q},
$$

we have

$$
F\left(b_{q, \phi(q), \psi(q)}\right) \subseteq F\left(a_{2 q-1}\right) \mid F\left(a_{2 q}\right),
$$

and we can find ar element $\gamma_{q+1} \in U$ such that

$$
\left\langle\gamma_{\phi(q)} \cdot \gamma_{q+1}\right\rangle \in F\left(a_{2 q-1}\right) \text { and }\left\langle\gamma_{q+1}, \gamma_{\psi(q)}\right\rangle \in F\left(a_{2 q}\right) .
$$

Using this together with (2), we infer from $\left(\Gamma_{3}\right)$ that

$$
\left\langle\gamma_{i}, \gamma_{q+1}\right\rangle \in F\left(b_{q+1, i, q+1}\right) \quad \text { for } i=0,1, \cdots, q .
$$

Hence, by $\left(\Gamma_{4}\right)$,

$$
\left\langle\gamma_{q+1}, \gamma_{j}\right\rangle \in F\left(b_{q+1, q+1, j}\right) \quad \text { for } j=0,1, \cdots, q .
$$

Finally, by $\left(\Gamma_{5}\right)$,

$$
\left\langle\gamma_{q+1}, \gamma_{q+1}\right\rangle \in F\left(b_{q+1, q+1, q+1}\right) .
$$

From (2)-(5) we see that (1) holds with $k=q+1$. Thus we find that $\gamma_{2}, \gamma_{3}$, $\cdots, \gamma_{n+1}$ can be so chosen that (1) holds for $k=1,2, \cdots, n+1$.

Applying (1) with $k=n+1, i=0$, and $j=1$, we see that $\langle\alpha, \beta\rangle \in F\left(b_{n+1,0,1}\right)$. Since this holds whenever $\langle\alpha, \beta\rangle \in F\left(a_{0}\right)$, we conclude that $F\left(a_{0}\right) \subseteq F\left(b_{n+1,0,1}\right)$ and hence $a_{0} \leqq b_{n+1,0,1}$. This completes the proof.

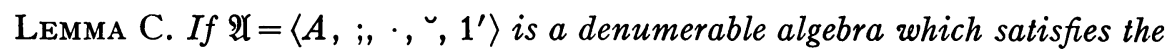
conditions (i)-(ix) and $(\Gamma)$, and if $d \in A$, then there exists a function $F$ which maps $\mathfrak{A}$ homomorphically onto an algebra of relations in such a way that, for every $x \in A$,

$$
F(d) \subseteq F(x) \text { if and only if } d \leqq x .
$$

Proof. We begin by deriving certain simple consequences from the conditions (i)-(ix). From (i)-(iii) it follows that the relation $\leqq$ partially orders $A$ and that any two elements $a, b \in A$ have the element $a \cdot b$ as their greatest lower bound. We shall show that, for every $a, b, c \in A$,

$$
\begin{aligned}
a^{\sim} \leqq b^{\sim} \text { if and only if } a \leqq b . \\
a \leqq b \text { implies that } a ; c \leqq b ; c . \\
b \leqq c \text { implies that } a ; b \leqq a ; c . \\
\\
1^{\prime \sim}=1^{\prime} . \\
1^{\prime} ; a=a .
\end{aligned}
$$




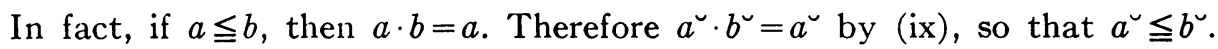
Conversely, if $a^{\sim} \leqq b^{\sim}$, then $a^{\sim \sim} \leqq b^{u}$ and hence $a \leqq b$ by (vii). Thus (1) holds. (2) is just a reformulation of (vi). If $b \leqq c$, then $b \subseteq c^{\sim}$ by (1) and therefore $b^{\sim} ; a^{\sim} \leqq c^{\sim} ; a^{\sim}$ by (2). Using (viii) we infer that $(a ; b)^{\sim} \leqq(a ; c)^{\sim}$, and a second application of (1) gives $a ; b \leqq a ; c$. This proves (3). Using successively (v), (vii), (viii), (v) and (vii), we find that

$$
1^{\prime \sim}=1^{\prime \sim} ; 1^{\prime}=1^{\prime \sim} ; 1^{\prime \sim}=\left(1^{\prime \sim} ; 1^{\prime}\right)^{\sim}=1^{\prime \sim}=1^{\prime}
$$

thus proving (4). Finally, by (vii), (viii), (4), (v) and (vii),

$$
1^{\prime} ; a=\left(1^{\prime} ; a\right)^{\sim}=\left(a^{\sim} ; 1^{\prime \sim}\right)^{\smile}=\left(a^{\lrcorner} ; 1^{\prime}\right)^{\smile}=a^{\lrcorner}=a,
$$

so that (5) also holds.

Assuming now that the hypothesis in condition $(\Gamma)$ is satisfied, consider a positive integer $k \leqq n$. We shall show that

$$
\begin{aligned}
b_{k, i, i} & \leqq 1^{\prime} & & \text { for } i=0,1, \cdots, k . \\
b_{k+1, i, j} & \leqq b_{k, i, j} & & \text { for } i, j=0,1, \cdots, k . \\
\left(b_{k, i, j}\right)^{-} & =b_{k, j, i} & & \text { for } i, j=0,1, \cdots, k .
\end{aligned}
$$

For $k=1,(6)$ holds by $\left(\Gamma_{1}\right)$. Next observe that if (6) holds for a given $k$ then, by $\left(\Gamma_{2}\right),(2)$ and $(5)$,

$$
b_{k+1, i, j} \leqq b_{k, i, i} ; b_{k, i, j} \leqq 1^{\prime} ; b_{k, i, j}=b_{k, i, j} .
$$

Thus, for any given $k,(6)$ implies (7). Furthermore, if (6) and (7) hold for a given $k$, we take $j=i$ in $(7)$ and infer that the formula $b_{k+1, i, i} \leqq 1^{\prime}$ holds for $i=0,1, \cdots, k$. Since, by $\left(\Gamma_{5}\right)$, this formula also holds for $i=k+1$, it follows that (6) holds with $k$ replaced by $k+1$. It is now easy to complete the proof of (6) and (7) by induction. Finally, (8) follows from $\left(\Gamma_{1}\right),\left(\Gamma_{2}\right),\left(\Gamma_{4}\right)$ and $\left(\Gamma_{5}\right)$ with the aid of (vii), (viii), (ix) and (4).

In the remainder of this proof, (i)-(ix) and (1)-(8) will be used freely without being explicitly referred to.

We next wish to define an infinite sequence $a_{0}, a_{1}, \cdots \in A$ with $a_{0}=d$, and two functions $\phi$ and $\psi$ on the positive integers, in such a way that the hypothesis in the condition $(\Gamma)$ holds for every positive integer $n$ and, furthermore, the following condition is satisfied:

For every $x, y \in A$, every positive integer $k$, and all natural numbers $i \leqq k$ and $j \leqq k$, if

$$
b_{k, i, j} \leqq x ; y,
$$

then there exists a positive integer $m>k$ such that

$$
\phi(m)=i, \psi(m)=j, a_{2 m-1}=x \text { and } a_{2 m}=y .
$$

For this purpose we arrange all the quadruples $\langle i, j, x, y\rangle$, where $i$ and $j$ are 
natural numbers and $x, y \in A$, into a sequence

$$
\left\langle p_{k}, q_{k}, u_{k}, v_{k}\right\rangle, \quad k=1,2, \cdots
$$

in such a way that each quadruple occurs infinitely many times in the sequence. For $k=1,2, \cdots$ let

$$
\begin{array}{lll}
\phi(k)=p_{k} \text { if } p_{k} \leqq k, & \phi(k)=k \text { if } p_{k}>k, \\
\psi(k)=q_{k} \text { if } q_{k} \leqq k, & \psi(k)=k \text { if } q_{k}>k .
\end{array}
$$

Assuming that $a_{0}, a_{1}, \cdots, a_{2 n-2}$ have been selected, and the elements $b_{k, i, j}$ with $k=1,2, \cdots, n$ and $i, j=0,1, \cdots, k$ thereby determined according to $\left(\Gamma_{1}\right)-\left(\Gamma_{5}\right)$, we let

$$
\begin{array}{lll}
a_{2 n-1}=u_{n} & \text { and } & a_{2 n}=v_{n} \text { if } b_{n, \phi(n), \psi(n)} \leqq u_{n} ; v_{n}, \\
a_{2 n-1}=b_{n, \phi(n), \psi(n)} & \text { and } \quad a_{2 n}=1^{\prime} \text { if } b_{n, \phi(n), \psi(n)} \leqq u_{n} ; v_{n} .
\end{array}
$$

It is easy to check that the sequence $a_{0}, a_{1}, \cdots$ and the functions $\phi$ and $\psi$ have the required properties.

We now define the function $F$ : For each $x \in A$ we let $F(x)$ be the relation consisting of all ordered pairs $\langle i, j\rangle$ of natural numbers such that $b_{n, i, j} \leqq x$ for some positive integer $n$ with $i \leqq n$ and $j \leqq n$.

Clearly $F(x \cdot y) \subseteq F(x) \cap F(y)$ for every $x, y \in A$. On the other hand, if $\langle i, j\rangle \in F(x) \cap F(y)$, then there exist positive integers $k$ and $m$, greater than or equal to $i$ and $j$, such that $b_{k, i, j} \leqq x$ and $b_{m, i, j} \leqq y$. Letting $n$ be the larger of the integer $k$ and $m$, we infer that $b_{n, i, j} \leqq x \cdot y$ and therefore $\langle i, j\rangle \in F(x \cdot y)$. Thus

$$
F(x \cdot y)=F(x) \cap F(y) \text { for every } x, y \in A .
$$

Now suppose $\langle i, j\rangle \in F(x ; y)$. Then $b_{k, i, j} \leqq x ; y$ for some positive integer $k$ greater than or equal to $i$ and $j$. We can therefore find a positive integer $m>k$ for which (9) holds. Uskng $\left(\Gamma_{3}\right)$ and $\left(\Gamma_{4}\right)$, we infer that

$$
\begin{aligned}
& b_{m+1, i, m+1} \leqq b_{m+1, i, \phi(m)} ; a_{2 m-1}=b_{m+1, i, i} ; x \leqq 1^{\prime} ; x=x, \\
& b_{m+1, m+1, j} \leqq a_{2 m} ; b_{m+1, \psi(m), j} \leqq y ; b_{m+1, j, j} \leqq y ; 1^{\prime}=y,
\end{aligned}
$$

from which it follows that $\langle i, m+1\rangle \in F(x)$ and $\langle m+1, j\rangle \in F(y)$ and, consequently, $\langle i, j\rangle \in F(x) \mid F(y)$. Conversely, suppose $\langle i, j\rangle \in F(x) \mid F(y)$. Then there exists a natural number $p$ such that $\langle i, p\rangle \in F(x)$ and $\langle p, j\rangle \in F(y)$, and we can find positive integers $k$ and $m$ such that $k \geqq i, k \geqq p, m \geqq p, m \geqq j, b_{k, i, p} \leqq x$ and $b_{m, p, j} \leqq y$. Letting $n$ be the larger of the two integers $k$ and $m$, we infer that $b_{n, i, p} \leqq x$ and $b_{n, p, j} \leqq y$, and it follows by $\left(\Gamma_{2}\right)$ that

$$
b_{n+1, i, j} \leqq b_{n, i, p} ; b_{n, p, j} \leqq x ; y .
$$

Consequently $\langle i, j\rangle \in F(x ; y)$. We have shown that

$$
F(x ; y)=F(x) \mid F(y) \quad \text { for every } x, y \in A .
$$


Using (8) we see that

$$
F\left(x^{5}\right)=F(x)^{-1} \quad \text { for every } x \in A,
$$

and from (v) and (11) we infer that

$$
F(x) \mid F\left(1^{\prime}\right)=F(x) \quad \text { for every } x \in A .
$$

It follows from (10)-(13) that $F$ maps $\mathfrak{A}$ homomorphically onto an algebra of relations. Finally suppose $x \in A$ and $F(d) \subseteq F(x)$. Since $d=a_{0}=b_{1,0,1}$, we have $\langle 0,1\rangle \in F(x)$. Hence $b_{n, 0,1} \leqq x$ for some positive integer $n$. But, according to the condition $(\Gamma), d \leqq b_{n, 0,1}$, and we conclude that $d \leqq x$. This completes the proof.

Lemma D. Any denumerable algebra $\mathfrak{A}=\left\langle A, ;, \cdot, \sim, 1^{\prime}\right\rangle$ which satisfies the conditions (i)-(ix) and $(\Gamma)$ is isomorphic to an algebra of relations.

Proof. By Lemma $\mathrm{C}$ we can associate with each element $d \in A$ a function $F_{d}$ which maps $\mathfrak{A}$ homomorphically onto an algebra of relations over some set $U_{d}$ in such a way that, for every $x \in A$,

$$
F_{d}(d) \subseteq F_{d}(x) \text { if and only if } d \leqq x .
$$

We can clearly choose the sets $U_{d}$ in such a way that they are pairwise disjoint. For each $x \in A$ define $F(x)$ to be the set-theoretic sum of all the relations $F_{d}(x)$ with $d \in A$. It is easy to check that $F$ maps $\mathfrak{A}$ homomorphically onto an algebra of relations. Furthermore, for any $x, y \in A$, if $F(x)=F(y)$, then $F_{d}(x)=F_{d}(y)$ for every $d \in A$. Hence, in particular, $F_{x}(x) \subseteq F_{x}(y)$ and $F_{y}(y)$ $\subseteq F_{y}(x)$. But this implies that $x \leqq y$ and $y \leqq x$ so that, finally, $x=y$. Thus $F$ is an isomorphism.

Proof of Theorem 1. Let $K$ be the class of all those algebras which are isomorphic to algebras of relations, and let $L$ be the class of all those algebras which satisfy the conditions (i)-(ix) and $(\Gamma)$. By Lemma A, $K$ is an arithmetic class in the wider sense, and by Lemma $B, K$ is a subclass of $L$. Now suppose $\mathfrak{A} \in L$. Then any finite subsystem $\mathfrak{B}$ of $\mathfrak{U}$ generates a denumerable subalgebra $\mathbb{C}$ of $\mathfrak{A}$. Clearly $\mathfrak{S} \in L$, and it follows by Lemma $\mathrm{D}$ that $\mathfrak{S} \in K$. Thus every finite subsystem of $\mathfrak{A}$ can be isomorphically embedded in a system in $K$, whence it follows by Henkin [2, Theorem 1 ], that $\mathfrak{A}$ itself can be so embedded. Since every subalgebra of an algebra in $K$ is itself in $K$, this implies that $\mathfrak{A} \in K$. Thus $K=L$, as was to be shown.

3. Applications. We shall now show how Theorem 1 yields a characterization of all those lattices which are isomorphic to lattices of commuting equivalence relations. As a further application we characterize the class of all those relation algebras which have a weak representation, i.e. an isomorphic representation in terms of binary relations where all the operations except Boolean addition (and complementation) have their natural meaning. We 
also give an example of a commutative integral relation algebra which does not have a weak representation.

It is well known that if two equivalence relations $R$ and $S$ over a set $U$ commute under relation-theoretic multiplication, i.e., if $R|S=S| R$, then $R \mid S$ is also an equivalence relation over $U$ and coincides with the lattice sum of $R$ and $S$ in the lattice of all equivalence relations over $U$. It follows that if $Q$ is a nonempty family of equivalence relations over $U$ with the property that $R \cap S \in Q$ and $R|S=S| R \in Q$ for every $R, S \in Q$, then the system

$$
\langle a, \mid, \cap\rangle
$$

is a lattice. Such a lattice we call a lattice of commuting equivalence relations.

It is not difficult to show (see e.g. Birkhoff $[1$, p. 85$]$ ) that every lattice of commuting equivalence relations is modular. While there do exist modular lattices which are not isomorphic to lattices of commuting equivalence relations, the only known examples are of a more or less pathological nature, such as the lattice of all subspaces of a non-Arguesian projective plane (Jónsson [4]). It is therefore natural to try to characterize all those lattices for which such a representation does exist.

THEOREM 2. In order for a lattice $\mathfrak{A}=\langle A,+, \cdot\rangle$ to be isomorphic to a lattice of commuting equivalence relations it is necessary and sufficient that the following condition be satisfied:

$\left(\Gamma^{\prime}\right)$ Suppose $n$ is a positive integer, $a_{0}, a_{1}, \cdots, a_{2 n}, z \in A$ and, for each positive integer $k \leqq n, \phi(k)$ and $\psi(k)$ are natural numbers with $\phi(k) \leqq k$ and $\psi(k) \leqq k$. Let

$$
b_{1,0,1}=b_{1,1,0}=a_{0}, \quad b_{1,0,0}=b_{1,1,1}=z,
$$

and for $k=1,2, \cdots, n$ and $i, j=0,1, \cdots, k$ let

$$
\begin{aligned}
b_{k+1, i, j} & =\coprod_{p \leqq k}\left(b_{k, i, p}+b_{k, p, j}\right), \\
b_{k+1, i, k+1} & =b_{k+1, k+1, i}=\left(b_{k+1, i, \phi}(k)+a_{2 k-1}\right) \cdot\left(b_{k+1, i, \psi(k)}+a_{2 k}\right), \\
b_{k+1, k+1, k+1} & =z .
\end{aligned}
$$

With these notations, if $b_{k, \phi(k), \psi(k)} \leqq a_{2 k-1}+a_{2 k}$ for $k=1,2, \cdots, n$, then $a_{0}$ $\leqq b_{n+1,0,1}$.

Proof. First assume that $\mathfrak{A}$ has a zero element 0 . Considering the algebra

$$
\mathfrak{H}^{\prime}=\langle A,+, \cdot, \breve{,} 0\rangle
$$

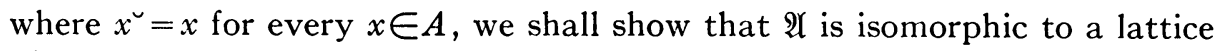
of commuting equivalence relations if and only if $\mathfrak{H}^{\prime}$ is isomorphic to an algebra of relations.

In fact, if $F$ maps $\mathfrak{A}$ isomorphically onto a lattice of commuting equivalence relations $\langle Q, \mid, \cap\rangle$, then the system $\langle Q, \mid, \cap,-1, F(0)\rangle$ is clearly an 
algebra of relations, and is the isomorphic image of $\mathfrak{A}^{\prime}$ under $F$. Conversely, if $F$ maps $\mathfrak{H}^{\prime}$ isomorphically onto an algebra of relations $\langle a, \mid, \cap,-1, I\rangle$, then $F$ maps $\mathfrak{A}$ isomorphically onto the system $\langle Q, \mid, \cap\rangle$. Since $x+x=x=x$ for every $x \in A$, we have $R \mid R=R=R^{-1}$ for every $R \in Q$. Thus the members of $Q$ are equivalence relations. Since $x \leqq y+x$ for every $x, y \in A$, we have $R \subseteq S \mid R$ for every $R, S \in Q$, which shows that all the members of $a$ have a common domain $U$. Since the operation + is commutative, we have $R \mid S$ $=S \mid R$ for every $R, S \in a$. Thus $\langle Q, \mid, \cap\rangle$ is in fact a lattice of commuting equivalence relations.

The algebra $\mathfrak{A}^{\prime}$ clearly satisfies the conditions (i)-(ix) of Theorem 1 (with + and 0 taking the place of ; and $1^{\prime}$ ). Therefore $\mathfrak{A}^{\prime}$ is isomorphic to an algebra of relations if and only if it satisfies the condition $(\Gamma)$. But since $x^{\circ}=x$ for every $x \in A$, this is clearly equivalent to the special case of our present condition $\left(\Gamma^{\prime}\right)$ in which $z=0$. However, if $\left(\Gamma^{\prime}\right)$ holds for $z=0$, it clearly holds for every $z \in A$, for if we replace $z$ by 0 in the construction of the elements $b_{k, i, j}$, these elements will be decreased, thus replacing the hypothesis $b_{k, \phi(k), \psi(k)} \leqq a_{2 n-1}+a_{2 n}$ by a weaker hypothesis and the conclusion $a_{0} \leqq b_{n, 0,1}$ by a stronger conclusion.

We now drop the hypothesis that $\mathfrak{A}$ has a zero element. If $\mathfrak{A}$ is isomorphic to a lattice of commuting equivalence relations, then so is every sublattice of $\mathfrak{A}$, in particular the sublattice generated by the elements $a_{0}, a_{1}, \cdots, a_{2 n}, z$. Inasmuch as every finitely generated lattice has a zero element, it follows by the first part of the proof that the condition $\left(\Gamma^{\prime}\right)$ must be satisfied. Conversely, assume that $\left(\Gamma^{\prime}\right)$ holds. We construct a new lattice $B=\langle B,+, \cdot\rangle$ by adjoining a zero element 0 to $\mathfrak{A}$. It is then easy to see that every finitely generated sublattice of $\mathfrak{B}$ is isomorphic to a sublattice of $\mathfrak{A}$, whence it follows that $\mathfrak{B}$ satisfies the condition $\left(\Gamma^{\prime}\right)$. Consequently, by the first part of the proof, $\mathfrak{B}$ is isomorphic to a lattice of commuting equivalence relations, and so is the sublattice $\mathfrak{A}$ of $\mathfrak{B}$. This completes the proof.

A relation algebra has been defined (see e.g. Jónsson-Tarski [5, Definition 4.1]) as an algebra

$$
\mathfrak{N}=\left\langle A,+, 0, \cdot, 1, ; 1^{\prime}, \breve{\gamma}\right\rangle
$$

such that $\langle A,+, 0, \cdot, 1\rangle$ is a Boolean algebra, the formulae

$$
x ;(y ; z)=(x ; y) ; z \text { and } x ; 1^{\prime}=1^{\prime} ; x=x
$$

hold for every $x, y, z \in A$, and the formulae

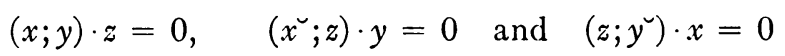

are equivalent for every $x, y, z \in A$. If, in particular, $A$ is a family of binary relations, 0 is the null-relation $\varnothing$, and the operations,$+ \cdot$, ; and coincide with set-theoretic addition, set-theoretic multiplication, relation-theoretic multiplication, and relation-theoretic conversion, then $\mathfrak{P}$ is called a proper 
relation algebra. By a representation of a relation algebra $\mathfrak{A}$ we mean a function which maps $\mathfrak{A}$ isomorphically onto a proper relation algebra, and $\mathfrak{A}$ is said to be representable if such a function $F$ exists. By a weak representation of $\mathfrak{A}$ we shall mean a function $F$ which maps $\mathfrak{A}$ isomorphically onto a relation algebra

$$
\langle\propto, \oplus, \varnothing, \cap, V, \mid, I,-1\rangle
$$

where $Q$ is a family of binary relations and $\varnothing, \cap, \mid,-1$ have their usual meaning, and we shall say that $\mathfrak{A}$ is weakly representable if there exists a function $F$ which is a weak representation of $\mathfrak{A}$. It is easy to see that a weak representation of $\mathfrak{A}$ is simply a function $F$ which maps the algebra $\left\langle A, ;, \cdot,, 1^{\prime}\right\rangle$ isomorphically onto an algebra of relations, and which has the additional property that $F(0)=\varnothing$.

'THEOREM 3. A relation algebra

$$
\mathfrak{H}=\left\langle A,+, 0, \cdot, 1, ; 1^{\prime},{ }^{\prime}\right\rangle
$$

is weakly representable if and only if it satisfies the condition $(\Gamma)$ of Theorem 1.

Proof. By Theorem 1, and in view of the preceding remark, it is sufficient to show that if there exists a function $F$ which maps the algebra $\mathfrak{H}^{\prime}$ $=\left\langle A, ;, \cdot,,^{\prime}\right\rangle$ isomorphically onto an algebra of relations, then there exists another function $G$ which also maps $\mathfrak{A}^{\prime}$ isomorphically onto an algebra of relations, and which has the additional property that $G(0)=\varnothing$. We let

$$
G(x)=F(x)-F(0) \quad \text { for every } x \in A .
$$

Then $G(0)=\varnothing$. Since $F(0) \subseteq F(x)$ for every $x \in A$, we see that $G$ is one-to-one. Since, clearly,

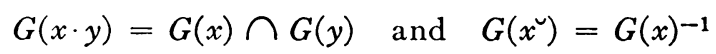

for every $x, y \in A$, it only remains to show that

$$
G(x ; y)=G(x) ; G(y) \quad \text { for every } x, y \in A .
$$

Observe that

$$
\begin{aligned}
G(x ; y) \cup F(0) & =F(x ; y)=F(x) \mid F(y) \\
& =[G(x) \cup F(0)] \mid[G(y) \cup F(0)] \\
& =[G(x) \mid G(y)] \cup[G(x) \mid F(0)] \cup[F(0) \mid G(y)] \cup[F(0) \mid F(0)] .
\end{aligned}
$$

Since

$$
\begin{aligned}
& F(0) \mid F(0)=F(0 ; 0)=F(0), \\
& G(x)|F(0) \subseteq F(x)| F(0)=F(x ; 0)=F(0), \\
& F(0)|G(y) \subseteq F(0)| F(y)=F(0 ; y)=F(0)
\end{aligned}
$$


it follows that

$$
G(x ; y) \cup F(0)=[G(x) \mid G(y)] \cup F(0) .
$$

By the definition of $G, G(x ; y) \cap F(0)=\varnothing$, and in order to prove (1) it is therefore sufficient to show that

$$
[G(x) \mid G(y)] \cap F(0)=\varnothing
$$

or, equivalently, that

$$
\left[G(x)^{-1} \mid F(0)\right] \cap G(y)=\varnothing .
$$

This last formula holds because

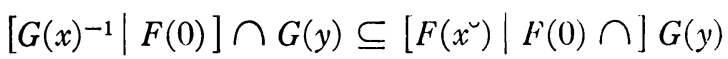

$$
\begin{aligned}
& =F(\check{x} ; 0) \cap G(y)=F(0) \cap G(y)=\varnothing,
\end{aligned}
$$

and the proof is therefore complete.

It is interesting to contrast this result with Theorem 4.22 of JónssonTarski [6], which states that every relation algebra has a representation wherein the operations + , ; and have their natural meaning. On the other hand, in Lyndon [7, p. 715] it is shown that there exist nonrepresentable relation algebras, and actually the algebra constructed there is not even weakly representable. We shall outline here the construction of another such algebra which satisfies certain rather strong additional conditions. Recall that a relation algebra is said to be integral if $x ; y=0$ always implies that $x=0$ or $y=0$. Excluding the trivial case in which $0=1$, we know (Jónsson-Tarski [6, Theorem 4.17]) that this holds if and only if $1^{\prime}$ is an atom of $A$.

THEOREM 4. There exists an integral relation algebra

$$
\mathfrak{i}=\left\langle A,+, 0, \cdot, 1, ; 1^{\prime}, \cup^{\prime}\right\rangle
$$

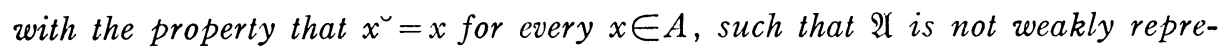
sentable.

Outline of proof. Given a relational system $\mathfrak{l}=\left\langle U, R, 1^{\prime}\right\rangle$ where $1^{\prime} \in U$ and $R$ is a ternary relation, we associate with $\mathfrak{U}$ an algebra

$$
\mathfrak{A}=\left\langle A,+, 0, \cdot, 1, ; 1^{\prime},{ }^{\sim}\right\rangle
$$

in such a way that $\langle A,+, 0, \cdot, 1\rangle$ is a complete atomistic Boolean algebra whose atoms are precisely the elements of $U$; for every $x, y \in A, x ; y$ is the Boolean sum of all atoms $w$ such that $\langle u, v, w\rangle \in R$ for some atoms $u \leqq x$ and

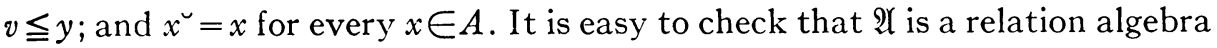
if and only if the following three conditions are satisfied:

(1) For every $x, y \in U,\left\langle x, 1^{\prime}, y\right\rangle \in R$ if and only if $x=y$.

(2) $\left\{\begin{array}{l}\text { For every } x, y, z \in U \text {, the conditions }\langle x, y, z\rangle \in R,\langle x, z, y\rangle \in R,\langle z, y, x\rangle \in R \\ \text { are equivalent. }\end{array}\right.$ 
(3) $\{$ For every $x, y, z, u, v \in U$, if $\langle x, y, u\rangle \in R$ and $\langle u, z, v\rangle \in R$, then there exists $w \in U$ such that $\langle y, z, w\rangle \in R$ and $\langle x, w, v\rangle \in R$.

Given a relational system $\mathfrak{U}_{0}=\left\langle U_{0}, R_{0}, 1^{\prime}\right\rangle$, an extension $\mathfrak{U}=\left\langle U, R, 1^{\prime}\right\rangle$ of $\mathfrak{U}_{0}$ will be called admissible if the following condition holds:

For any four distinct elements $x, y, z, u \in U_{0}$, if there exists $v \in U_{0}$ such that $\langle x, y, v\rangle \in R_{0}$ and $\langle z, u, v\rangle \in R_{0}$, then there exists no $t \in U-U_{0}$ such that $\langle x, y, t\rangle \in R$ and $\langle z, u, t\rangle \in R$. We wish to show that

I. If $\mathfrak{U}_{0}=\left\langle U_{0}, R_{0}, 1^{\prime}\right\rangle$ is a relational system satisfying (1) and (2), then there exists an admissible extension $\mathfrak{U}=\left\langle U, R, 1^{\prime}\right\rangle$ of $\mathfrak{U}_{0}$ which satisfies (1), (2) and (3).

First we prove a weaker statement: that

II. If $\mathfrak{U}_{0}=\left\langle U_{0}, R_{0}, 1^{\prime}\right\rangle$ satisfies (1) and (2) and if $x, y, z, u, v \in U_{0}$ are such

$$
\langle x, y, u\rangle \in R_{0} \quad \text { and } \quad\langle u, z, v\rangle \in R_{0}
$$

then there exists an admissible extension $\mathfrak{U}=\left\langle U, R, 1^{\prime}\right\rangle$ of $\mathfrak{H}_{0}$ satisfying (1) and (2), such that

$$
\langle y, z, w\rangle \in R \quad \text { and } \quad\langle x, w, v\rangle \in R \quad \text { for some } w \in U .
$$

In fact, if (5) holds with $\mathfrak{U}=\mathfrak{U}_{0}$ and $R=R_{0}$, then we may take $\mathfrak{U}=\mathfrak{U}_{0}$. Observe in particular, that this will be the case if one of the elements $x, y, z, v$ is $1^{\prime}$, for we may then take $w$ to be $v, z, y$, or $x$ respectively. In the alternative case we let $U$ be a set obtained by adjoining to $U_{0}$ a single element $w \notin U_{0}$, and let $R$ be the relation obtained by adjoining to $R_{0}$ the ordered triples $\langle y, z, w\rangle,\langle x, w, v\rangle,\left\langle w, 1^{\prime}, w\right\rangle$, and all ordered triples obtained from these by permuting the terms. It is not hard to see that the relational system $\mathfrak{u}$ $=\left\langle U, R, 1^{\prime}\right\rangle$ is in fact an admissible extension of $\mathfrak{U}_{0}$ satisfying (1) and (2), and clearly (5) is also satisfied.

By a transfinite iteration of the embedding in II we obtain:

III. If $\mathfrak{H}_{0}=\left\langle U_{0}, R_{0}, 1^{\prime}\right\rangle$ is a relational system satisfying (1) and (2), then there exists an admissible extension $\mathfrak{U}=\left\langle U, R, 1^{\prime}\right\rangle$ of $\mathfrak{u}_{0}$, satisfying (1) and (2), such that for every $x, y, z, u, v \in U_{0}$, (4) implies (5).

Finally, by an infinite iteration of the embedding in III, we arrive at I.

We are now ready to construct the algebra called for in the theorem. Taking a non-Arguesian projective plane $P$, let $U_{0}$ be the set consisting of all the points of $P$ and of one additional element $1^{\prime}$, and let $R_{0}$ be the ternary relations whose elements are all ordered triples $\langle x, y, z\rangle$ where $x, y$ and $z$ are distinct collinear points of $P$, and all ordered triples $\left\langle x, x, 1^{\prime}\right\rangle,\left\langle x, 1^{\prime}, x\right\rangle$, $\left\langle 1^{\prime}, x, x\right\rangle$ with $x \in U_{0}$. It is easy to show that the relational system $\mathfrak{U}_{0}$ $=\left\langle U_{0}, R_{0}, 1^{\prime}\right\rangle$ satisfies (1) and (2). Hence there exists an admissible extension $\mathfrak{U}=\left\langle U, R, 1^{\prime}\right\rangle$ of $\mathfrak{U}_{0}$ which satisfies (1), (2) and (3), and we can associate with 1 l a relation algebra $\mathfrak{A}$ in the manner described at the beginning of the proof. By definition, $x^{-}=x$ for every $x \in A$, and from the fact that $1^{\prime}$ is an atom of 
$\mathfrak{A}$ it follows that $\mathfrak{A}$ is integral. We complete the proof by showing that $\mathfrak{A}$ is not weakly representable.

Suppose $\mathfrak{A}$ has a weak representation $F$. Consider two triangles of $P$ which are centrally perspective but not axially perspective. Let the vertices of the triangles be $a_{0}, a_{1}, a_{2}$ and $b_{0}, b_{1}, b_{2}$, respectively, let the center of perspectivity be $z$, and for $i=0,1,2$ let $c_{i}$ be the point of intersection of the sides opposite to $a_{i}$ and to $b_{i}$. Using the fact that $\mathfrak{l}$ is an admissible extension of $\mathfrak{l}_{0}$, we see that

$$
c_{i}=\left(a_{j}, a_{k}\right) \cdot\left(b_{j} ; b_{k}\right) \quad \text { and } \quad z=\left(a_{i} ; b_{i}\right) \cdot\left(a_{j} ; b_{j}\right)
$$

for $i, j, k=0,1,2$ with $i \neq j \neq k \neq i$. Therefore, given any ordered pair $\langle\alpha, \beta\rangle \in F\left(c_{0}\right)$, we can find elements $\gamma$ and $\delta$ such that

$$
\langle\alpha, \gamma\rangle \in F\left(a_{1}\right), \quad\langle\gamma, \beta\rangle \in F\left(a_{2}\right), \quad\langle\alpha, \delta\rangle \in F\left(b_{1}\right), \quad\langle\delta, \beta\rangle \in F\left(b_{2}\right) .
$$

Thus

$$
\langle\gamma, \delta\rangle \in\left[F\left(a_{1}\right) \mid F\left(b_{1}\right)\right] \cap\left[F\left(a_{2}\right) \mid F\left(b_{2}\right)\right]=F(z) \subseteq F\left(a_{0}\right) \mid F\left(b_{0}\right),
$$

and there exists an element $\lambda$ such that

$$
\langle\gamma, \lambda\rangle \in F\left(a_{0}\right), \quad\langle\lambda, \delta\rangle \in F\left(b_{0}\right) .
$$

It follows that

$$
\langle\alpha, \lambda\rangle \in\left[F\left(a_{1}\right) \mid F\left(a_{0}\right)\right] \cap\left[F\left(b_{1}\right) \mid F\left(b_{0}\right)\right]=F\left(c_{2}\right)
$$

and, similarly, $\langle\lambda, \beta\rangle \in F\left(c_{1}\right)$. Consequently,

$$
\langle\alpha, \beta\rangle \in F\left(c_{2}\right) \mid F\left(c_{1}\right)=F\left(c_{2} ; c_{1}\right) .
$$

We have thus shown that $F\left(c_{0}\right) \subseteq F\left(c_{2} ; c_{1}\right)$, whence $c_{0} \leqq c_{2} ; c_{1}$. This, however, implies that $c_{0}, c_{1}$ and $c_{2}$ are collinear, contrary to our assumption that the two triangles are not axially perspective.

It does of course follow that the condition $(\Gamma)$ is not satisfied. Actually we could prove this directly, and use it to establish the nonexistence of a weak representation. In fact, take $n=3$, for the elements $a_{0}, a_{1}, \cdots, a_{6}$ in the condition $(\Gamma)$ take $c_{0}, a_{1}, a_{2}, b_{1}, b_{2}, a_{0}, b_{0}$, and let

$$
\phi(1)=\phi(2)=0, \phi(3)=2, \quad \psi(1)=\psi(2)=1, \psi(3)=3 .
$$

It then turns out that $b_{4,0,1}=c_{1}+c_{2}$, and the conclusion of $(\Gamma)$ would therefore yield $c_{0} \leqq c_{1}+c_{2}$, contrary to the fact that $c_{0}, c_{1}$ and $c_{2}$ are not collinear.

4. Unsolved problems. In conclusion we shall mention three open questions related to the results obtained here.

Problem 1. Can the infinite sets of axioms contained in the conditions $(\Gamma)$ and $\left(\Gamma^{\prime}\right)$ be replaced by finite sets of axioms? By sets of equations?

It would of course be particularly interesting if it should turn out that these conditions could be replaced by finite sets of identities. In this connec- 
tion we recall (Jónsson [3]) that a lattice $\mathfrak{A}=\langle A,+, \cdot\rangle$ is said to be Arguesian if it satisfies the following condition:

For every $a_{0}, a_{1}, a_{2}, b_{0}, b_{1}, b_{2} \in A$, if

$$
y=\left(a_{0}+b_{0}\right) \cdot\left(a_{1}+b_{1}\right) \cdot\left[\left(a_{0}+a_{2}\right) \cdot\left(b_{0}+b_{2}\right)+\left(a_{1}+a_{2}\right) \cdot\left(b_{1}+b_{2}\right)\right],
$$

then

$$
\left(a_{0}+b_{0}\right) \cdot\left(a_{1}+b_{1}\right) \cdot\left(a_{2}+b_{2}\right) \leqq a_{0} \cdot\left(a_{1}+y\right)+b_{0} \cdot\left(b_{1}+y\right) .
$$

In Jónsson [4] it is shown that every lattice of commuting equivalence relations is Arguesian. It is an open question whether, conversely, every Arguesian lattice is isomorphic to a lattice of commuting equivalence relations, but we know that this is the case for complemented lattices (Jónsson [3, Theorem 2.14]) as well as for lattices of dimension four or less (Jónsson [5, Result 5],- - the proof is unpublished).

PROBLem 2. Is every modular lattice isomorphic to a lattice of commuting equivalence elements of some relation algebra?

By an equivalence element of a relation algebra

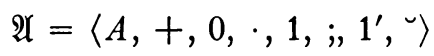

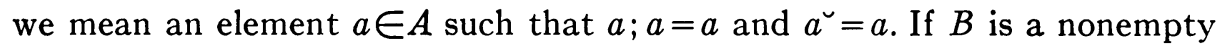
set of equivalence elements of $A$ with the property that, for every $a, b \in B$,

$$
1^{\prime} \leqq a, \quad a \cdot b \in B \text { and } a ; b=b ; a \in B
$$

then it is easy to check that the system $\langle B, ;, \cdot\rangle$ is a modular lattice. Such a lattice we call a lattice of commuting equivalence elements of $\mathfrak{A}$. The close connection between the representation problems for modular lattices and for relation algebras suggests that the solution to this problem may be affirmative.

Problem 3. Do there exist weakly representable relation algebras which are not representable?

Since Lyndon's characterization of representable relation algebras is considerably more involved than our condition $(\Gamma)$, a negative answer to this question would constitute an improvement of his result.

\section{BiBLIOGRAPHY}

1. G. Birkhoff, Lattice theory, rev. ed., Amer. Math. Soc. Colloquium Publications, vol. 25, New York, 1948.

2. L. Henkin, Some interconnections between modern algebra and mathematical logic, Trans. Amer. Math. Soc. vol. 74 (1953) pp. 410-427.

3. B. Jónsson, Modular lattices and Desargues' theorem, Math. Scand. vol. 2 (1954) pp. 295-314.

4. - - On the representation of lattices, Math. Scand. vol. 1 (1953) pp. 193-206.

5. - - Representations of lattices II, Bull. Amer. Math. Soc. vol. 60 (1954) p. 24.

6. B. Jonsson and A. Tarski, Boolean algebras with operators II, Amer. J. Math. vol. 74 (1952) pp. 127-162. 
7. R. Lyndon, The representation of relation algebras, Ann. of Math. vol. 51 (1950) pp. 707-729.

8. - The representation of relation algebras II, Ann. of Math. vol. 63 (1956) pp. 294307.

9. A. Tarski, Contributions to the theory of models, I, II, and III, Nederl. Akad. Wetensch. Proc. Ser. A. vol. 57 (1954) pp. 573-581, vol. 57 (1954) pp. 582-588 and vol. 58 (1955) pp. 56-64.

University OF MinNESOTA,

Minneapolis, MinN. 